\title{
Hot Streak Shaping and Migration in an Axial Turbine
}

\author{
Kai Regina, Altug M. Basol, Philipp Jenny, Anestis I. Kalfas ${ }^{1}$ and Reza S. Abhari \\ Laboratory for Energy Conversion \\ Department of Mechanical and Process Engineering \\ ETH Zurich, Switzerland \\ E-Mail: regina@lec.mavt.ethz.ch
}

\begin{abstract}
Hot streaks can cause local hot spots on the blade surfaces of high-pressure turbine stages, resulting in locally higher thermal loads. These local loads represent a potential source of blade life reduction and blade failure. The blade regions exposed to higher thermal loads are determined by the effect of the unsteady blade row interaction on the migration path of the hot streaks. In order to improve understanding of these effects an experimental study on the effect of shaping the inlet temperature distortion has been undertaken.

The experimental investigations have been performed in the axial turbine facility "LISA" at ETH Zurich. The test configuration consists of a one-and-1/2 stage, unshrouded, highly loaded axial turbine with a hot streak generator placed upstream of the first vane row. The latter is designed to provide different shapes of the inlet temperature distortion, as well as different circumferential and spanwise positions. The steady and unsteady aerodynamic effects are measured respectively with pneumatic probes and the in-house developed Fast Response Aerodynamic Probe (FRAP) technology. The unsteady thermodynamic effects are measured in a time resolved manner with the in-house developed Fast Response Entropy Probe (FENT). The time resolved measurements are made in planes at the inlet to the first vane row as well as downstream of it and downstream of the rotor.

The current paper presents the results of the first shaped hot streak injection and analyzes the mechanisms involved in the convection and the migration of the hot streak through the bladed rows.

The effect of the first stationary blade row on the path of the hot streak is explained by an analysis of the flow field and temperature field at the exit of the first nozzle guide vane row. Mixing and heat conduction as well as the unsteady effect of the downstream rotor cause the total temperature distortion to diminish thus generating a more uniform distribution.

The effect of the rotating blade row is shown with the flow field and the temperature field at the exit of the rotor. The measurements reveal a radial migration of the hot streak which is confined in circumferential direction by the pressure side of the rotor wake causing the fluid to partially go into the tip leakage vortex. Furthermore, at the suction side of the rotor blade the hot gases are confined in between the passage vortices of the row. The root mean square of the unsteady pressure signal acquired can be used for tracing the mixing process and losses showing the interaction of the hot streak with the secondary flow structures.
\end{abstract}

\footnotetext{
1 Department of Mechanical Engineering, Aristotle University of
} Thessaloniki, GR 54124 Thessaloniki, Greece

\section{INTRODUCTION}

With the goal of improving the efficiency of modern gas turbines, current design methods yield to a constantly increasing turbine entry temperature over the years. Measurement data acquired from gas turbines show combustor exit flows exhibiting strong radial and circumferential temperature non-uniformities which are termed as hot streaks. These temperature non-uniformities are a result of the circumferentially discrete fuel and dilution air injection in the combustor. As the hot streaks convect downstream through the turbine blade rows, the higher temperature regions locally increase the incident heat load on downstream components. This overheating can potentially overload the components and reduce their life resulting in significant risks or in damages in the worst case. Consequently, turbine components are often designed to withstand higher temperatures than the mean turbine entry temperature. Apart from that, the hot streaks have a different migration pattern within the bladed rows, generating additional secondary flows. A better understanding of thermally driven transport mechanisms induced by hot streaks has been the aim of several experimental and numerical investigations.

Among the first studies of inlet flow distortion effects on a stationary blade rows there is the work done by Munk and Prim [1]. In their work it was shown theoretically that the streamline pattern in a vane passage is not influenced by the presence of any variation in total temperature as long as the stagnation pressure distributions remain unchanged and the flow is steady. In a numerical study, Hermanson and Thole [2] have shown that the dominant parameter for end wall secondary flow generation in a turbine vane is the stagnation pressure distribution rather than the total temperature. This has also been confirmed by experimental studies on the transport mechanisms of combustor exit total pressure and total temperature profiles conducted in a high pressure turbine vane by Barringer et al. [3]. The resulting effects of non-uniform inlet temperatures on the heat transfer of high pressure vanes and end walls have been investigated by Povey et al. [4] both numerically and experimentally as well as experimentally by Barringer et al. [5] and Mathison et al. [6]. The combined effect of high turbulence and film cooling on the mixing and dispersion of a hot streak in a NGV was experimentally studied by Jenkins et al [7]. It was shown that turbulence effects and film cooling considerably reduce the hot spot peak temperatures.

The more complex effects of an inlet temperature distortion on rotating blade rows have been studied experimentally since the work by Butler et al. [8]. It was observed that the hot gases preferentially migrate towards the pressure side of the rotating blades. This is attributed to the fact that the hot gases travel with a higher velocity than the main flow since they have an elevated 
temperature but the same Mach number. Therefore, the hot gases arrive at the rotor with a higher incidence than the colder main flow resulting in the separation of hot and cold gases. This separating effect had also been reported by Kerrebrock and Mikolajczak [9] within their work on the transport of high temperature compressor rotor wakes through the downstream NGV row. The combination of hot streak and vane coolant injection in a turbine and their effects on the rotor unsteady flow field have been focus of both numerical investigations by An et al. [10] as well as of experimental work by Ong et al. [11].

The aim of the present work is to analyze the unsteady and thermally driven transport mechanisms resulting from an inlet temperature distortion generator. This analysis is based on a measurement campaign which was performed in a research turbine providing conditions representative for real engines. The asymmetrical shape of the hot streak with respect to the center of the first NGV passage is modelling the effect that the control of dilution air would have on the exit temperature field of a real combustor. A comparison with a previous work in the same turbine environment but with a circular hot streak gives insight into the effect of the hot streak shaping on the flow field. Further numerical investigations on the shaping of the non-uniform temperature inlet distortion with the same turbine have been reported in Basol et al [12].

\section{EXPERIMENTAL METHOD}

The experimental investigation was performed in the research axial turbine facility "LISA" in the Laboratory for Energy Conversion at ETH Zurich. The one-and-1/2 stage unshrouded turbine is representative for a high work, cooled turbine. Details of the turbine design were presented by Behr et al. [13] but the most relevant features are described below. The first NGV has non-axisymmetric end wall contouring at hub and tip whereas the two subsequent rows have conventional axisymmetric end walls. More details on the design of the contoured end walls and its measured impact on the flow field can be found in Germain et al [14] and Schuepbach et al. [15] respectively. Additionally, an inlet temperature distortion generator, which had been designed and presented by Jenny et al. [16], was used upstream of the first NGV.

\section{Research Turbine Facility}

The research turbine facility consists of a quasi-closed air loop which includes a single stage radial compressor, a two-stage water to air heat exchanger and a calibrated venturi nozzle for mass flow measurements. Upstream of the turbine section there is a 3-m-long flow conditioning stretch in order to ensure a homogenous flow field. In addition, the flow undergoes an acceleration ahead of the turbine to reduce the significance of any remaining flow non-uniformities from upstream. At the exit of the turbine section the air loop is open to atmosphere. A DC generator absorbs the turbine's power and controls the rotational speed with an indicated accuracy of $\pm 0.02 \%$ ( $\pm 0.5 \mathrm{rpm})$. The water to air heat exchanger controls the inlet total temperature $T_{0, \text { in }}$ to an accuracy of $\pm 0.3 \mathrm{~K}$. A torquemeter measures the torque on the rotor shaft. Since the compressor pressure ratio is limited to $\Pi_{c, \max }=1.5$ it is necessary to add a tandem deswirl vane arrangement to recover the static pressure at the exit of the second NGV back to the ambient level in order to reach the intended turbine pressure ratio of $\Pi_{1.5}=1.65$. The unshrouded turbine has a nominal tip gap of $1 \%$ of the span and the variation of the tip gap between different assemblies is less than $1 \%$ of the tip gap ensuring good repeatability. At the exit of the first NGV the flow is compressible with an average exit Mach number of 0.53 .

\section{Operating Conditions}

During the measurements the turbine 1.5 stage total-to-static pressure ratio is kept constant at $\Pi_{1.5}=1.65$. The entry and the hot streak temperatures were kept constant to allow an accurate comparison between measurements carried out on different days. In order to account for the change in ambient pressure on different measurement days, the pressures are non-dimensionalized by the respective inlet stagnation pressure. The main operating parameters are summarized in Table 1 .

Table 1: Operating conditions and geometrical characteristics

\begin{tabular}{lll}
\hline \hline$\Pi_{1.5}$ & $1.65 \pm 0.4 \%$ & {$[-]$} \\
$T_{0, \text { in }}$ & $314 \pm 0.3$ & {$[\mathrm{~K}]$} \\
$\frac{\dot{m} \sqrt{T_{0, \text { in }}}}{p_{0, \text { in }}}$ & $151.6 \pm 0.2$ & {$\left[\frac{\mathrm{kg} \cdot \mathrm{K}^{1 / 2}}{\mathrm{~s} \cdot \mathrm{bar}}\right]$} \\
$\frac{N}{\sqrt{T_{0, \text { in }}}}$ & $2.54 \pm 0.05$ & {$\left[\frac{\mathrm{rps}}{\mathrm{K}^{1 / 2}}\right]$} \\
Mach nr (S1 in / S1 ex / R ex) & $0.14 / 0.52 / 0.24$ & \\
Reynolds $\mathrm{nr}(\mathrm{S} 1 / \mathrm{R} / \mathrm{S} 2)$ & $7.1 / 3.8 / 5.1$ & {$\left[\times 10^{5}\right]$} \\
Blade count (S1 / R / S2) & $36 / 54 / 36$ & {$[-]$} \\
Aspect ratio (S1 / R / S2) & $0.87 / 1.17 / 0.82$ & {$[-]$} \\
\hline \hline
\end{tabular}

\section{Generator of inlet temperature distortion}

By means of the inlet temperature distortion generator, one single hot streak was introduced into the main flow approximately 3.5 NGV1-axial-chords upstream of the first NGV row. The mass flow of the hot streak was adapted such that its stagnation pressure and Mach number match the same level as the free stream. The variable position of the hot streak injector tube was fixed at midspan and midpitch. Its asymmetric shape was designed to deliver a non-uniform distribution which has different extents on the ends of the hot streak as it approaches the first NGV. In Figure 1 the designed shape of the injector tube is depicted.

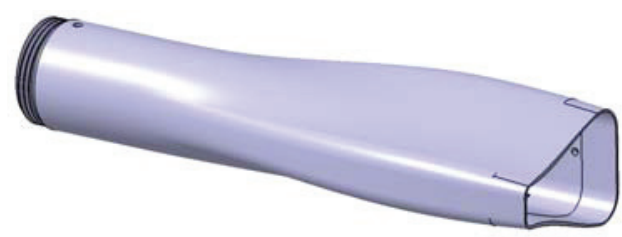

Figure 1: Shape of the hot streak injector tube

In the analyzed case the side of the hot streak which was closer to the pressure side of the NGV had a greater radial extent than the opposite side. In a real engine such a shape of the inlet temperature non-uniformities can be achieved by means of dilution air control in the liner of the combustor.

\section{Measurement Technology}

The unsteady flow field measurements were performed using a Fast Response Aerodynamic Probe (FRAP), which had been developed at the LEC at ETH Zurich. Details on the FRAP probe and measurement technique are presented in depth in Kupferschmied et al. [17] and Pfau et al. [18]. The FRAP probe is capable of capturing the unsteady flow features up to frequencies of $48 \mathrm{kHz}$ based on measurements including the total and static pressures, flow yaw and pitch angles and Mach number. The frequency bandwidth of the temperature is limited to a frequency of $10 \mathrm{~Hz}$. However, the influence of the measured temperature on the velocity is judged to be very modest. The FRAP probe has a $1.8 \mathrm{~mm}$ tip diameter and is equipped with two miniature silicon piezo-resistive sensors. The probe is operated in a virtual-4-sensor mode to measure 3-dimensional, time-resolved flow properties. The data is acquired at a sampling rate of $200 \mathrm{kHz}$ over a period of time of $2 \mathrm{~s}$. The postprocessing is done for three consecutive rotor pitches. The sampling rate resolves 82 points in the rotor relative frame of reference. The typical measurement uncertainties obtained with the FRAP probe for a calibration range of $\pm 24^{\circ}$ for the yaw angle and $\pm 20^{\circ}$ for the pitch angle are given in Table 2 . The relative uncertainties of the total and static pressures are given as a 
percentage of the dynamic head.

Table 2: Relative uncertainty bandwith of the FRAP probe

\begin{tabular}{llll}
\hline \hline Yaw angle & Pitch angle & $\mathrm{P}_{\mathrm{t}}$ & $\mathrm{P}_{\mathrm{s}}$ \\
$0.24^{\circ}$ & $0.36^{\circ}$ & $1 \%$ & $1.2 \%$ \\
\hline \hline
\end{tabular}

The unsteady temperature field was measured using the Fast Response Entropy Probe (FENT) developed in-house at the LEC at ETH Zurich. Details on the FENT probe and measurement technique are presented in depth in Mansour et al. [19], [20].The probe has a tip diameter of $1.8 \mathrm{~mm}$ and consists mainly of two parts. First, a miniature piezo-resistive sensor is used to obtain the time-resolved total and static pressures. Second, two thin film gauges with a thickness of approximately $200 \mathrm{~nm}$ are operated as resistance thermometers for the acquisition of the unsteady total temperature. With the knowledge of the unsteady total temperature and the unsteady total pressure, the unsteady relative entropy can be derived.

The thin film gauges have a serpentine shape and cover a rectangular area of $1.77 \mathrm{~mm} \times 0.85 \mathrm{~mm}$. The radial extent of the thin film gauges represents approximately $2.5 \%$ of the blade span and is the minimum spatial resolution in the measurement grid. The measurements carried out with the FENT probe have a radial spacing of $1.125 \mathrm{~mm}$ where a radial clustering was done and 2.25 $\mathrm{mm}$ elsewhere. In the data postprocessing a phase-locking is done and coincident phase-locked measurements of total pressure and total temperature are used for the determination of the unsteady relative entropy. The measurement bandwidth of the FENT probe is $48 \mathrm{kHz}$ and the data is acquired at a sampling frequency of $200 \mathrm{kHz}$ over a period of time of $2 \mathrm{~s}$ as is for the FRAP probe. The typical relative uncertainties achieved with the FENT probe are given in Table 3 .

Table 3: Relative uncertainty bandwith of the FENT probe

\begin{tabular}{lll}
\hline \hline $\mathrm{P}_{\mathrm{t}}$ & $\mathrm{T}_{\mathrm{t}}$ & $\Delta \mathrm{s}$ \\
$0.1 \%$ & $2.5 \%$ & $2.51 \%$ \\
\hline \hline
\end{tabular}

\section{Measurement Planes}

The measurement data was acquired at three different axial locations in the turbine test facility by traversing the probe in radial and circumferential direction. The geometry and the relative positions of the NGVs to the inlet temperature distortion generator as well as the positions of the traversing planes, $\mathrm{S} 1$ in (A), S1 ex (B) and R ex (C) are shown in Figure 2.

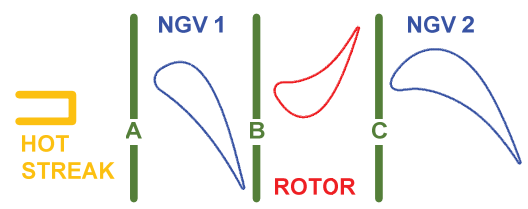

Figure 2: Illustration of geometry and measurement planes

The spatial resolution of the measurement grid at the traverse planes S1 ex and R ex consisted of 45 radial and 41 circumferential points covering one NGV1 pitch. At the traverse plane S1 in the measurement grid consisted of 45 radial and 37 circumferential points, only covering $90 \%$ of the NGV1 pitch. The circumferential points were equally spaced whereas the radial points were clustered near the end walls and in the region of the hot streak.

\section{RESULTS AND DISCUSSION}

In the following section the time resolved measurement data of the flow field and the temperature field are presented. The analysis will focus particularly on the thermally driven transport mechanisms highlighting the interaction between both flow field and temperature field. The discussions will start with the effect of the NGV on the convection of the hot streak and then continue with the unsteady effects of the rotor on the convection and migration pattern of the hot streak through the blade row.

\section{NGV Inlet Flow Field}

In Figure 3 the time averaged normalized total temperature measured with the FRAP probe at the inlet plane to the first NGV is depicted.

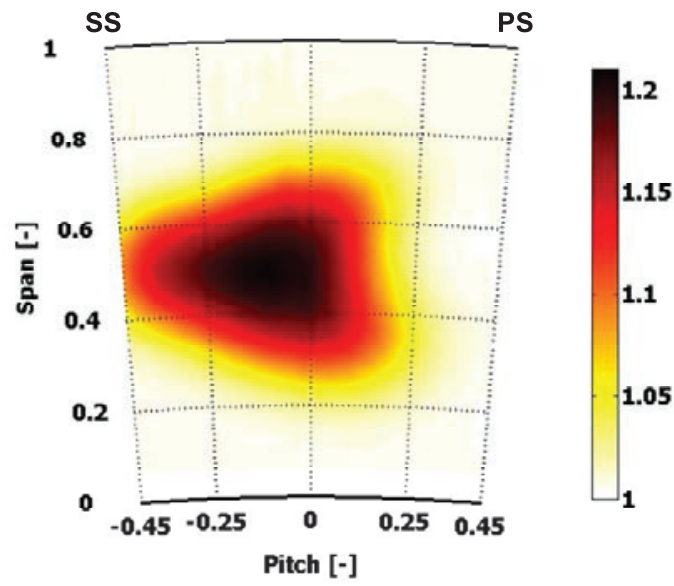

Figure 3: Time averaged normalized total temperature at the traverse plane $\mathrm{S} 1$ in $[-]$

The figure shows that the hot streak has the designed shape and the intended location at midspan. Furthermore, it can be seen that the temperature ratio in the core of the hot streak reaches the value of 1.21 , which corresponds to the total temperature ratio immediately at the exit of the injector tube. From that location, the hot streak travels approximately 3.5 NGV1-axial-chords until it reaches the traverse plane S1 in and ends having the depicted shape. Within this distance the hot gases of the hot streak have already initiated the mixing process with the main flow as can be seen by the lower total temperatures at the periphery of the hot streak. In Figure 4, the time averaged and circumferentially mass averaged normalized total temperature distribution along the span reveals the same initiated mixing process.

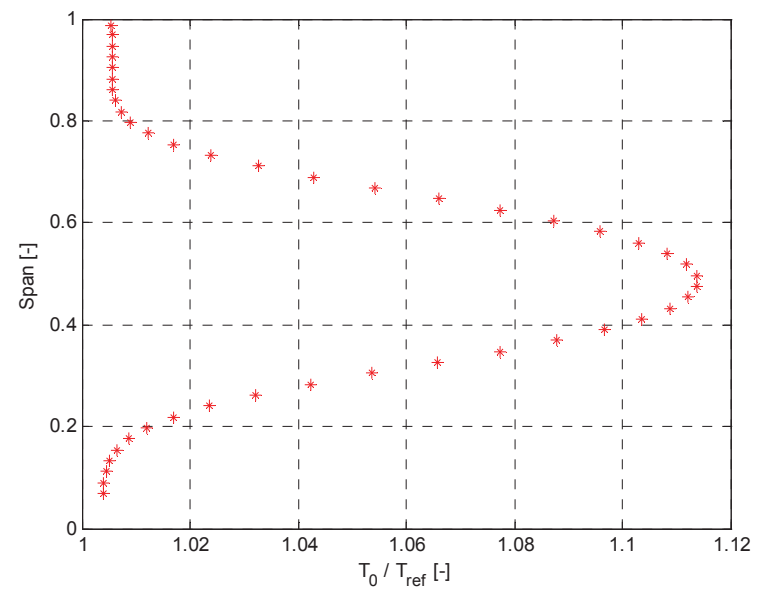

Figure 4: Time averaged and circumferentially mass averaged normalized total temperature at the traverse plane $\mathrm{S} 1$ in

The turbulence intensity at the inlet plane to the turbine was measured with the FRAP probe and the level reaches values up to $11 \%$ in the core region of the hot spot, whereas in the main stream of the flow the value is in the order of $1 \%$. The high turbulence intensity is enhancing the mixing out of the hot streak with the main stream.

Furthermore, the distribution in Figure 4 shows that the center of 
the hot streak is still located at $50 \%$ span although a strong mixing is ongoing.

\section{NGV Exit Flow Field}

The major effect of the NGV 1 on the convection of the hot spot can be highlighted by a comparison of the time averaged and circumferentially mass averaged radial distributions of the stagnation temperature at the inlet and exit traverse planes of the NGV1, see Figure 5.

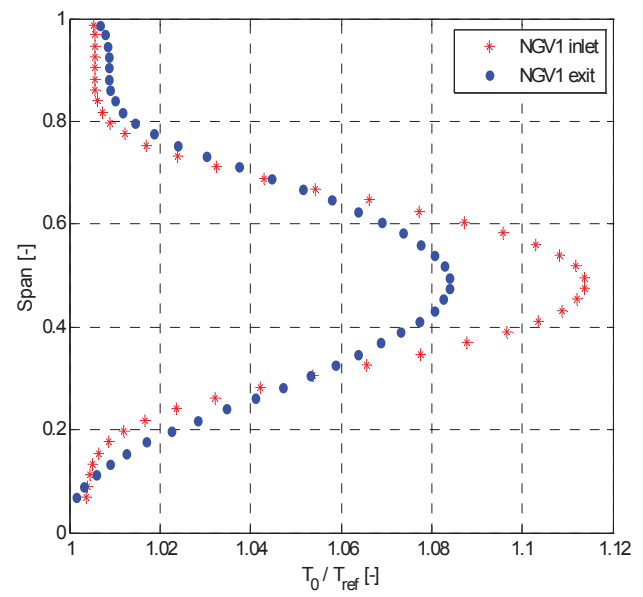

Figure 5: Time averaged and circumferentially mass averaged normalized total temperature at the traverse planes S1 in and S1 ex

First, it can be identified that the location of the center of the hot spot remains unchanged at $50 \%$ span height. Second, it can be seen that the mixing process has continued throughout the strong acceleration across the $\mathrm{NGV}$, since the peak total temperature is reduced and since the distribution becomes wider in radial direction.

A more detailed comparison can be done after analyzing the time averaged total temperature field shown in Figure 6.

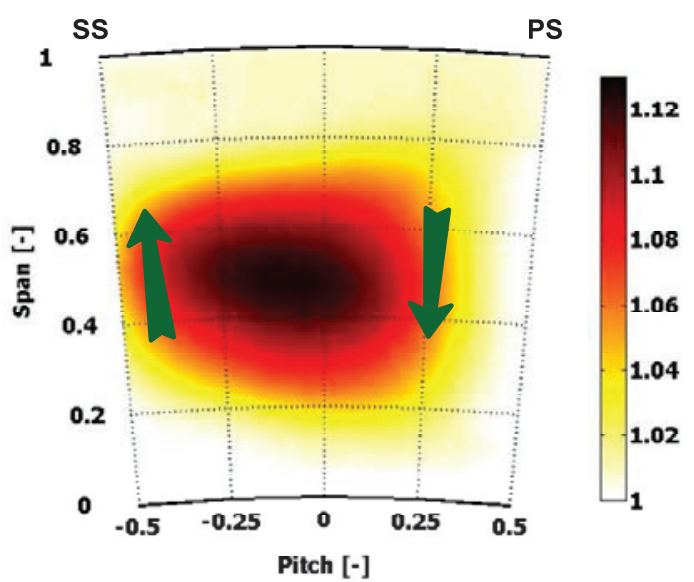

Figure 6: Time averaged normalized total temperature at the traverse plane S1 ex [-]

After convection through the NGV 1, the core region of the hot spot has a normalized total temperature of 1.13 , which corresponds to a reduction from NGV inlet to exit of approximately $7 \%$. In terms of temperature differences from the hottest gases to the main fluid this drop corresponds to a maximum unsteady temperature difference of $65 \mathrm{~K}$ at the inlet plane to the NGV1 to $42 \mathrm{~K}$ at the exit plane. This yields to a reduction of total temperature difference by $38 \%$ confirming the strong mixing.

Also, a strong attenuation in the circumferential non-uniformities of the hot streak can be observed. This can be seen by the change in shape as compared to the initial shape. Especially the side of the hot streak which initially had a smaller radial extent and which has passed closer to the suction side of the NGV experiences a stronger alteration than the opposite side. This indicates that the hot gases on the suction side of the NGV have initiated a radial migration towards the casing. The distribution of pitch angles in this region, which are depicted in Figure 7, also confirms this migration showing positive pitch angles on the suction side of the blade and negative pitch angles on the pressure side of the blade.

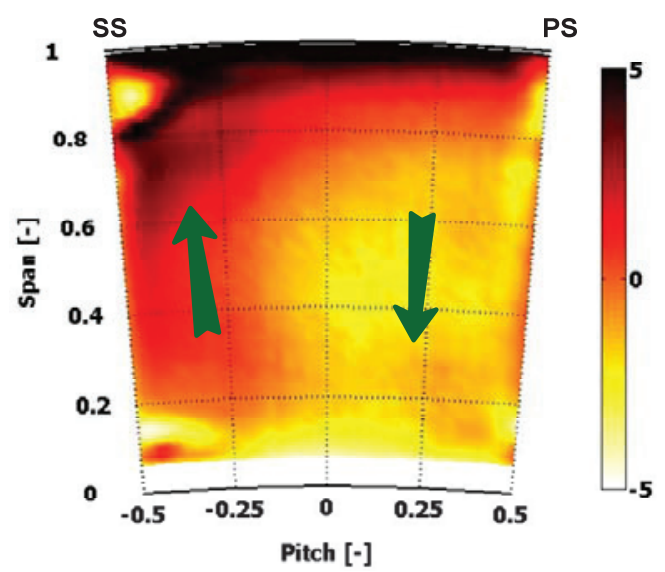

Figure 7: Time averaged pitch angle at the traverse plane S1 ex $\left[^{\circ}\right]$

According to the studies of Munk and Prim [1] a change in the streamline pattern in a vane passage should not be expected in a steady flow as long as the total pressure distribution remains unchanged. Therefore, an analysis of the time resolved temperature field at the traverse plane S1 ex reveals the unsteady nature of the temperature field caused by the upstream effect of the unsteady rotor potential field. In Figure 8 there is a series of field plots of the total temperature at the exit plane of the NGV 1 for six different equally spaced rotor blade passing periods. a) $\mathrm{t} / \mathrm{T}=0.01$

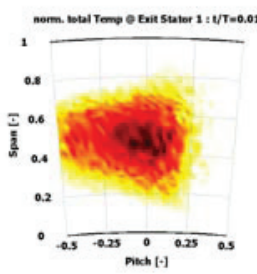

c) $\quad t / T=0.33$

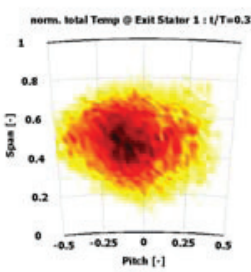

e) $t / T=0.67$

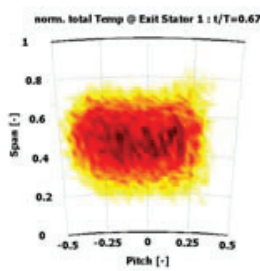

b) $\mathrm{t} / \mathrm{T}=0.16$

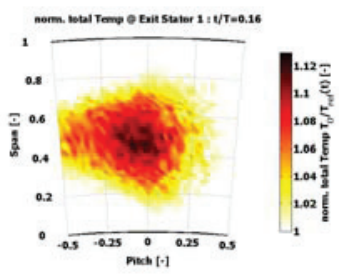

d) $t / T=0.50$
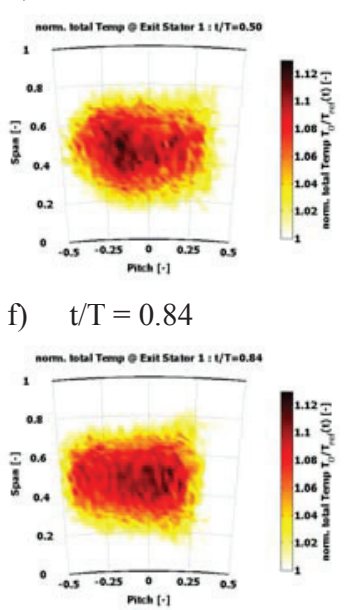

f) $t / T=0.84$

Figure 8: Normalized total temperature for different blade passing periods at the traverse plane S1 ex [-]

From figure 8 it can be observed that the gases with the highest 
temperatures in the core of the hot streak travel approximately $25 \%$ of the NGV1 pitch in circumferential direction while these gases at the same time also travel in radial direction by approximately $5 \%$ of the span height.

This effect, caused by the entrainment of the hot spot into the rotor potential field, was also observed and explained by Jenny et al. [16] The unsteady wobbling effect is responsible for the resulting blurred time-averaged shape of the hot streak as was shown in Figure 6.

\section{$\underline{\text { Rotor Exit Flow Field }}$}

As a next step the unsteady flow field at the traverse plane located at the exit of the rotor will be analyzed in the following in order to highlight the convection and migration of the hot streak through the rotating blade row.

The root mean square (rms) value of the random part of the total pressure signal is an appropriate indicator for tracking the hot streak downstream of the bladed row of the turbine. The rms is an indicator of turbulence intensity by which the hot streak can be identified since it has a distinct level than the rest of the fluid. Furthermore it can be used for identifying the hot streak downstream of the rotor since this value remains unaffected by the extraction of work from the fluid done by the rotor.

A comparison of the rms data of the present experimental campaign with the data of a previous experimental study done by Behr et al [13] at the same operating conditions but without inlet temperature distortion is shown in Figure 9, where the pitch-time-diagrams of the rms value are depicted.
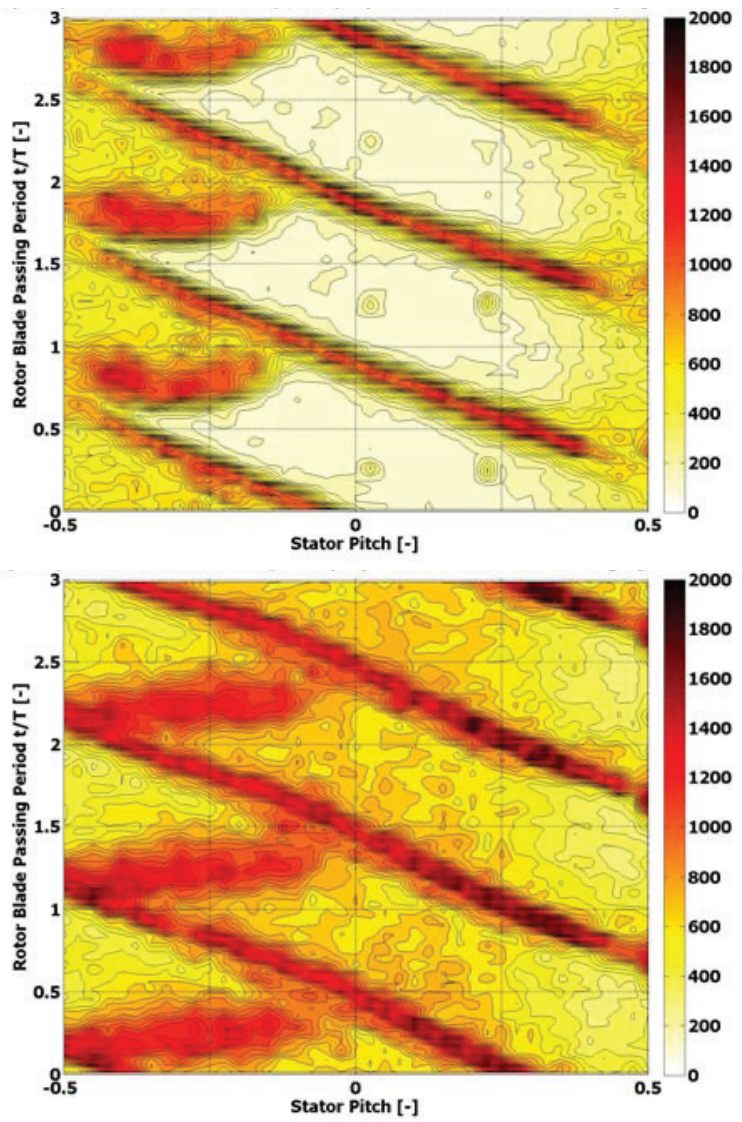

Figure 9: Pitch-time-diagrams of the rms value at midspan at the traverse plane R1 ex for the case without (top) and with (bottom) hot streak $[\mathrm{Pa}]$

The comparison of the two plots in Figure 9 shows a region of increased $\mathrm{rms}$ in the case with the hot streak which is confined in between the rotor wakes clearly identifying the location of the hot streak. Inside of the rotor passage, the level and the extent of the region of high rms is greater closer to the pressure side of the rotor wake, showing that the hot streak is coming preferably attached to the pressure side of the blade, which agrees well with the results of the previous measurement campaign with the circular hot streak [16] as well as with the observations of Kerrebrock and Mikolajczak [9].

A more detailed analysis can be done if the span-pitch contour plots of the rms value are also taken into consideration, see Figure 10.

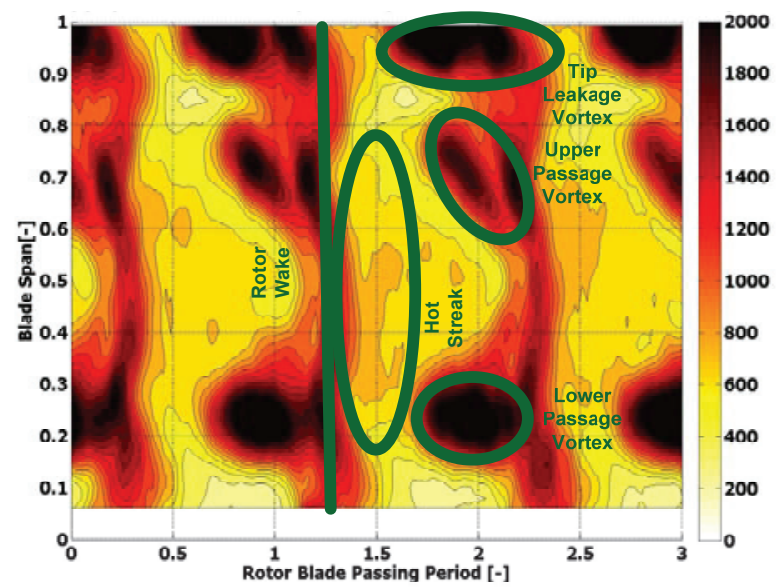

Figure 10: Span-time diagram of the rms value at the traverse plane $\mathrm{R} 1 \mathrm{ex}$ in the absolute frame of reference $[\mathrm{Pa}]$

The diagram in Figure 10 confirms again that there is a region of higher rms values which comes attached to the pressure side of the rotor wake. A further comparison with the time-resolved temperature distribution shall be done with the same type of diagram for the normalized total temperature at the exit of the rotor, see Figure 11. It shows the FENT-measurements at the same circumferential location as the FRAP-measurements of Figure 10.

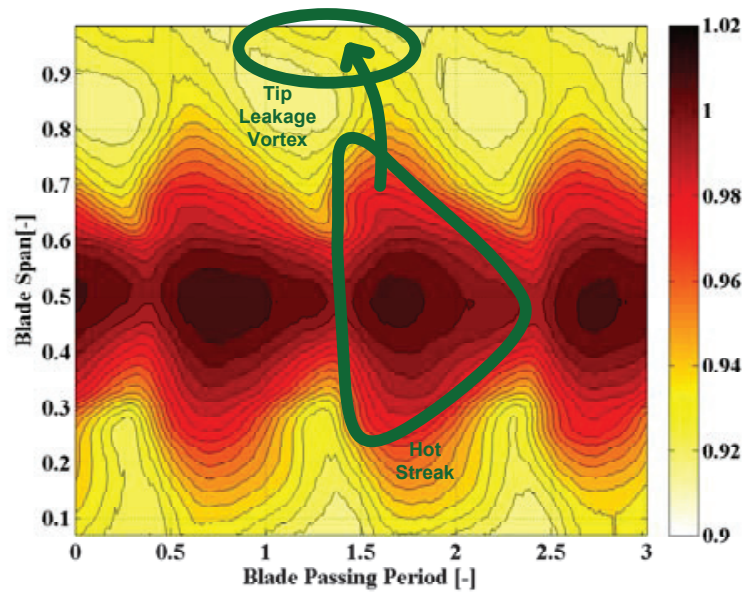

Figure 11: Span-time diagram of the normalized absolute total temperature at the traverse plane R1 ex in the absolute frame of reference [-]

The comparison of Figure 11 with Figure 10 confirms the radial migration of the hot streak towards hub and tip at the pressure side of the blade. Furthermore it can be identified that the hot gases partially go into the tip leakage vortex, which can be seen in Figure 10 and Figure 11 at $100 \%$ span and $0.3,1.3$ and 2.3 blade passing periods. Furthermore it can be seen that the hot gases do not penetrate into the high rms regions caused by the upper passage vortex, which is confined between $60 \%$ and $80 \%$ span height approximately. The same pattern applies also for the high rms region induced by the lower passage vortex, whose core can be identified to be at approximately $25 \%$ span.

The measured distribution of time averaged and circumferentially 
mass averaged normalized total temperatures at the inlet and the exit of the rotor row are presented in Figure 12, where the values have been normalized by the main stream temperature of the corresponding traverse plane respectively.

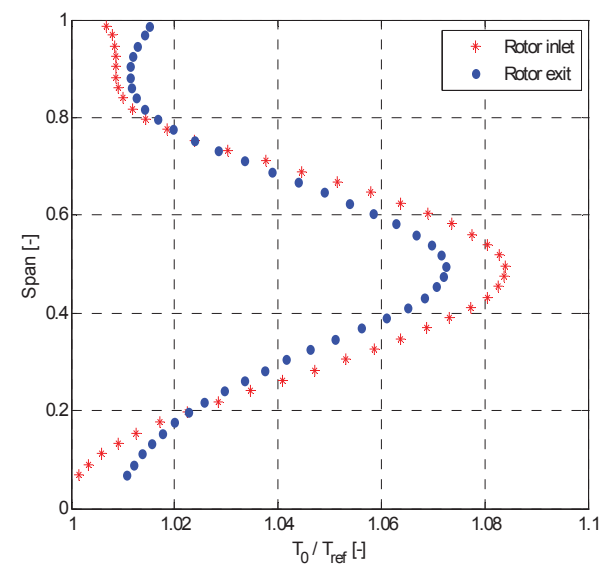

Figure 12: Time averaged and circumferentially mass averaged normalized absolute total temperature at the traverse planes S1 ex and $\mathrm{R} 1$ ex [-]

The distribution depicted in Figure 12 confirms the radial migration of the hot streak yielding a profile which is slightly wider in radial direction. Furthermore the region of the interaction between the tip leakage vortex and the hot streak can be seen with the already mentioned elevated total temperature since the fluid in the vortex has not contributed to work extraction in the same extent as the rest of the fluid and since hot gases have migrated into this region.

The comparison of the averaged distributions across the rotor also shows that the peak temperature of the hot streak remains unchanged at $50 \%$ span.

\section{CONCLUSIONS}

This paper presents combined time resolved measurements of both unsteady pressure as well as unsteady temperature acquired in a one-and-1/2 stage highly loaded research axial turbine with an unshrouded rotor using the Fast Response Aerodynamic Probe (FRAP) and the Fast Response Entropy Probe (FENT) developed at the LEC at ETH Zurich. The thermal effect of the unsteady blade row interactions on the migration and transport mechanisms of hot streaks was measured and analyzed throughout the first turbine stage. The hot spot was generated such that it matches non-dimensional parameters of real gas turbines at a peak temperature ratio of 1.21. The asymmetric shape of the hot streak is modelling the effect of dilution air control from a combustor liner on its resulting temperature field.

Confirming previous findings on the same turbine environment with a circular hot streak shape, the current measurements at the exit of the first NGV reveal to be strongly influenced by the downstream rotor unsteady potential field. The latter causes a wobbling of the hot streak in the circumferential direction with a frequency equal to the rotor blade passing frequency. While travelling in circumferential direction these gases also travel slightly into the radial direction. This migration is initiated already upstream of the rotor and is characterized by an outward migration on the suction side of the NGV 1 and an inward migration on the opposite side. This thermally induced secondary flow results in the initial asymmetry of the hot streak shape to be considerably attenuated. This bidirectional migration yields to the radial location of the center of the hot streak not being affected and remaining at $50 \%$ span across the first vane row.

Also confirming the previous measurements with the circular hot streak, in the current measurements the hot spot arrives with a higher incidence and velocity to the rotor, thus inducing a separation of the hot and cold gases inside of the rotor passage. This results to the hot gases being attached to the pressure surface of the rotor blade. Furthermore, the hot gases continue the migration in both radial directions inside of the rotor passage. The time resolved measurements show that part of the hot streak gases merges with the tip leakage vortex causing an increased heat load on the blade tip region. In addition to the previous measurements with the circular hot streak, the presented measurements also show that the hot streak is confined in between and does not penetrate those regions of the rotor passage, which are dominated by the passage vortices. However, these thermally driven flow patterns inside the rotor do not affect the radial location of the peak temperature inside of the core of the hot streak, which remains unchanged still at $50 \%$ span.

The present measurements with the asymmetrically shaped hot streak confirm the flow patterns which were identified in the previous measurement campaign with a circular hot streak and the same turbine environment. However, due to the shape of the hot streak its migration appears to be initiated at an earlier stage while convecting through the turbine. In the present case the peak temperature of the hot streak at the rotor exit is located at midspan while for the previous campaign with the circular hot streak it had been identified below $47 \%$ span. Therefore, the comparison shows how the resulting radial heat load distributions approaching components downstream of a combustor can be controlled by means of shaping the inlet temperature distortion to the turbine. In a real engine this can be achieved by controlling the dilution air injection in a combustor liner.

\section{ACKNOWLDGEMENTS}

The authors thank MTU Aero Engines for the permission to use their end wall contouring design.

\section{REFERENCES}

[1] Munk, M. and Prim, R. C., 1947, "On the Multiplicity of Steady Gas Flows Having the Same Streamline Pattern", Proceedings of the National Academy of Sciences 33(1), pp. 137-141

[2] Hermanson, K. S. and Thole, K. A., 2002, "Effect of Nonuniform Inlet Conditions on Endwall Secondary Flows", Journal of Turbomachinery, Vol. 124, pp. 623-631

[3] Barringer, M. D., Thole, K. A., Polanka, M. D., Clark, J. P. and Koch, P. J., 2009, "Migration of Combustor Exit Profiles Through High Pressure Turbine Vanes", Journal of Turbomachinery, Vol. 131

[4] Povey, T., Chana, K. S., Jones, T. V. and Hurrion, J., 2007, "The Effect of Hot-Streaks on HP Vane Surface ans Endwall Heat Transfer: An Experimental and Numerical Study", Journal of Turbomachinery, Vol. 129, pp. 32-43

[5] Barringer, M. D., Thole, K. A. and Polanka, M. D., 2009, “An Experimental Study of Combustor Exit Profile Shapes on Endwall Heat Transfer in High Pressure Turbines", Journal of Turbomachinery, Vol. 131

[6] Mathison, R. M., Haldeman, C. W. and Dunn, M. G., 2010 , "Aerodynamics and Heat Transfer for a Cooled One and One-Half Stage High-Pressure Turbine - Part III: Impact of Hot Streak Characteristics on Blade Row Heat Flux", Proceedings of ASME Turbo Expo 2010, ASME-GT2010-23855

[7] Jenkins, S., Varadarajan, K., Bogard, D. G., 2004, “The Effects of High Mainstream Turbulence and Turbine Vane Film Cooling on the Dispersion of a Simulated Hot Streak", Journal of Turbomachinery, Vol. 126, pp. 203-211

[8] Butler, T. L., Sharma, O. P., Joslyn, H. D. and Dring, R. P., 1989, "Redistribution of an Inlet Temperature Distortion in an Axial Flow Turbine Stage", Journal of Propulsion, Vol. 5, No. 1, pp. 64-71

[9] Kerrebrock, J. L., Mikolajczak, A. A., 1970, „Intra-Stator Transport of Rotor Wakes and Ist Effect on Compressor 
Performance“, Journal of Engineering for Power, pp. 359-368

[10] An, B.-T., Liu, J.-J., Jiang, H.-D., 2009, „Combined Unsteady Effects of Hot Streak an Trailing Edge Coolant Ejection in a Turbine Stage", Proceedings of ASME Turbo Expo 2009, ASME-GT2009-59473

[11] Ong, J., Miller, R. J., 2008, "Hot Streak and Vane Coolant Migration in a Downstream Rotor", Proceedings of ASME Turbo Expo 2008, ASME-GT2008-50971

[12] Basol, A. M., Regina, K., Kalfas, A. I., Abhari, R. S., 2011, "Integrated Combustor - Turbine Design for Improved Aerothermal Performance: Effect of Dilution Air Control", Proceedings of ASME Turbo Expo 2011, ASME-GT2011-46354

[13] Behr, T., Kalfas, A. I. and Abhari, R. S., 2007, "Unsteady Flow Physics and Performance of a One-and-1/2-Stage Unshrouded High Work Turbine", Journal of Turbomachinery, 129(2), pp. 348-359

[14] Germain, T., Nagel, M., Raab, I., Schuepbach, P. and Abhari, R. S., 2008, „Improving Efficiency of a High Work Turbine Using Non-Axisymmetric Endwalls: Part I - Endwall Design and Performance, Proceedings of ASME Turbo Expo 2008, ASME-GT2008-50469

[15] Schuepbach P., Abhari, R. S., Rose, M. G., Germain, T., Raab, I., Gier, J., 2008, „Improving Efficiency of a High Work Turbine Using Non-Axisymmetric Endwalls: Part I Time-Resolved Flow Physics, Proceedings of ASME Turbo Expo 2008, ASME-GT2008-50470
[16] Jenny, P., Lenherr, C., Kalfas, A., Abhari, R. S., 2010, „Effect of Hot Streak Migration on Unsteady Blade Row Interaction in an Axial Turbine", Proceedings of ASME Turbo Expo 2010, ASME-GT2010-23034

[17] Kupferschmied, P., Kopperl, O., Gizzi, W. P. and Gyarmathy, G., „Time Resolved Flow Measurements With Fast Aerodynamic Probes in Turbomachinery", Meas. Sci. Technol. 11 , pp. 1036-1054

[18] Pfau, A., Schlienger, J., Kalfas, A. I. and Abhari, R. S., „Unsteady 3-Dimensional Flow Measurement Using a Miniature Virtual 4 Sensor Fast Response Aerodynamic Probe (FRAP)“, Proceedings of ASME Turbo Expo 2003, ASME-GT2003-38128

[19] Mansour, M., Chokani, N., Kalfas, A. I. and Abhari, R. S., 2008, "Time-Resolved Entropy Measurements Using a Fast Response Entropy Probe", Meas. Sci. Technol. 19

[20] Mansour, M., Chokani, N., Kalfas, A. I. and Abhari, R. S., 2008, "Unsteady Entropy Measurements in a High Speed Radial Compressor", Journal of Engineering for Gas Turbines and Power, Vol. 130(2) 